\section{Continuando el camino}

\section{Continuing the road}

Cien años de desarrollo intenso e ininterrumpido de estudio y diseminación de conocimiento a favor del cuidado de la salud integral de niños y adolescentes, han sembrado en cada uno de los miembros de la Sociedad Argentina de Pediatría principios y valores que facilitan los desafíos.

Hay acuerdos básicos que tienen que ver con la formación, con el contacto con los maestros, con la tradición y sobre todo con la revalidación diaria frente a una comunidad que espera del pediatra respuestas adecuadas a sus necesidades, el acompañamiento en la crianza de sus hijos, una mirada amplia, responsable y de conocimiento.

Aspirar a la máxima equidad y universalidad para que todos los niños y adolescentes del país tengan derecho a estos cuidados ha sido desde siempre la motivación que lleva a la SAP a la búsqueda e implementación de recursos, de gestiones ante organismos públicos y privados, nacionales e internacionales que faciliten a los pediatras el acceso al conocimiento, espacios de reflexión e interacción, caminos que han colocado a la pediatría del país en un lugar reconocido y respetado.

Sostener y profundizar esta tarea compleja y centenaria, es el desafío.

Y ha sido un desafío para una sociedad científica abrir un campo de intercambio y análisis de la situación en que los pediatras desarrollan su labor en cada punto del país.

La encuesta SAP UNICEF, realizada en el universo de los pediatras de la Sociedad Argentina de Pediatría, es un importante aporte y brinda un elemento valioso para el análisis de la problemática del Ejercicio profesional, y la generación de acciones que apunten a mejorarlo.

Los múltiples empleos como consecuencia de las bajas remuneraciones, las diferencias de género y el incremento de la violencia en los ámbitos del ejercicio de la profesión son, probablemente, las conclusiones más significativas, pero la riqueza de la información obtenida es mucho más amplia y sugiere la complejidad de nuestra realidad.

Asimismo, el $64 \%$ de los pediatras manifiesta estar satisfecho con el ejercicio de su profesión, sustentando esta afirmación en la posibilidad de desarrollar su vocación.

Uno de los datos que surgen de manera nítida es la multiplicidad de empleos: la mayoría (70\%) trabaja en más de un lugar y dentro de este grupo algunos tienen 3 y hasta 4 trabajos, situación que se fundamenta en la necesidad de conseguir una remuneración razonable. Más del 65\% se desem- peña en el ámbito oficial, aunque sólo un pequeño porcentaje lo hace en forma exclusiva.

Como es sencillo de deducir, esto conspira contra las posibilidades de dedicar espacios a las actividades formativas, los momentos de reflexión y discusión, imprescindibles para el desarrollo personal y profesional.

Esto queda claramente explicitado en las respuestas vinculadas con el tiempo libre, que fue considerado insuficiente por la gran mayoría de los encuestados; buena parte del mismo es dedicado a la familia y un porcentaje mucho menor a actividades de esparcimiento.

Esta atomización en la actividad laboral afecta también la producción de los grupos de trabajo y la formación de equipos interdisciplinarios, que necesitan de tiempo para consolidar su funcionamiento.

La carga asistencial predomina sobre otros aspectos igualmente valiosos en el ejercicio de la profesión, lo cual se pone de manifiesto en el escaso número de quienes efectúan algún actividad de investigación.

Por otra parte, las sensaciones de cansancio y agotamiento verificadas son un elemento negativo de suma importancia que repercute en la calidad de la atención que reciben los pacientes, y casi seguramente en el aumento de las posibilidades de cometer errores.

Otro aspecto de marcada significación son las diferencias de género evidenciadas: en una actividad en la que hay una mayoría creciente de mujeres, se observa que ocupan menos cargos de gestión, tienen ingresos más bajos y son más frecuentemente víctimas de distinto tipo de violencia tanto en el entorno laboral como en el ejercicio de la profesión.

Probablemente esta situación está en consonancia con la que existe en la sociedad en general, pero lo cierto es que requiere producir acciones que corrijan una injustificable diferencia.

Al explorar las situaciones de violencia, pudo objetivarse que prácticamente la mitad de los encuestados sufrieron algún tipo de maltrato verbal o psicológico de parte de los pacientes, y un porcentaje menor $(5 \%)$, aunque no menos preocupante, agresión física directa, hechos que son más frecuentes en los grupos etarios más jóvenes probablemente porque son quienes hacen más guardias, y en las mujeres.

Estas circunstancias determinan una seria interferencia en la atención de la salud, imposibi- 
litando una adecuada relación médico paciente, y produciendo desaliento, aumento del estrés y planteos de abandono de la profesión.

El fenómeno de la violencia laboral ha sido reconocido en los últimos años como un problema universal, que adquiere cada vez mayores dimensiones.

"La violencia en el lugar de trabajo -sea física o psicológica-se ha convertido en un problema mundial que atraviesa las fronteras, los contextos de trabajo y los grupos de profesionales.

Si bien la violencia laboral afecta prácticamente a todos los sectores y categorías de trabajadores, el sector de atención de la salud corre grave riesgo. En este sector puede ser hasta la cuarta parte de la violencia que tiene lugar en el trabajo" (Directrices Marco para afrontar la violencia laboral en el sector de la salud - OMS OIT Ginebra 2002).

El abordaje de este problema debe ser sistemático, con la participación del estado, la sociedad y las instituciones de salud, tanto en lo que hace a medidas de fondo para disminuir la incidencia como en la elaboración de leyes y procedimientos para atenuar sus consecuencias.

Pero también existe un importante rol que deben jugar las Sociedades Científicas, que deben actuar en la formación del médico para prevenir y enfrentar estas situaciones, evaluar la posibilidad de introducir en los mecanismos de acreditación de instituciones la existencia de protocolos, y colaborar en la difusión hacia la comunidad.

También debe destacarse que más del $40 \%$ de los encuestados refieren haber sido víctimas de violencia verbal y/o psicológica en su entorno laboral, lo cual genera consecuencias en el desempeño y en el confort en el desarrollo del trabajo. Justamente, la existencia de un clima de trabajo agradable ha sido uno de los factores de satisfacción expresados.

Hecha una apretada síntesis de algunos de los aspectos interrogados, es necesario destacar que el $64 \%$ de los pediatras se manifestó satisfecho con su ejercicio profesional, y la mayoría sustentó esta opinión en la posibilidad de realizarse desarrollando su vocación.

Por supuesto que, si bien auspicioso, esto no invalida las crecientes dificultades que la profesión médica en general experimenta, muchas de las cuales quedan expuestas en los resultados discutidos previamente.

La vocación es el motor imprescindible que moviliza nuestras acciones pero corre el riesgo de declinar en la medida que las condiciones en que deben desarrollarse se tornan cada vez más difíciles, como lo demuestra la disminución de postulantes para algunas especialidades, lo cual abre serios interrogantes para el futuro.

En el convencimiento de que la sociedad en general debe conocer estas realidades y jugar un papel importante en las decisiones en salud, estos datos fueron difundidos en conferencia de prensa con amplia repercusión en todo el país.

Por otra parte, el amplio detalle que surge de esta encuesta permite proyectar un trabajo local y participativo, por lo que la SAP encara junto con Unicef la realización de un proyecto itinerante que convoque en cada Región a quienes, desde un análisis intersectorial y desde distintos niveles de decisión, puedan generar cambios efectivos a las situaciones desfavorables e iniciativas de fortalecimiento en donde se hayan alcanzado aspectos satisfactorios.

Son pasos en el camino que estamos decididos a recorrer para hacer efectiva y digna la privilegiada tarea de cuidar a nuestros chicos.

Dr. Gustavo Cardigni Presidente Sociedad Argentina de Pediatría http:/ /dx.doi.org/10.5546/aap.2012.282

\section{El comienzo de un trascendente cambio en Archivos Argentinos de Pediatría}

\section{The beginning of a transcendent change in Archivos Argentinos de Pediatría}

Cuando Archivos ingresó en Medline, en julio de 2008, en el párrafo final del editorial de agosto transmitía una reflexión acerca de este acontecimiento, de la cual resumo algunas frases: "Finalmente, deseo manifestar que es muy probable que el logro alcanzado signifique un hito y estemos ante un momento trascendente en la historia de la revista. También es muy probable que represente un significativo aporte para la pediatría del país y que el ingreso al Medline permita obtener otros múltiples beneficios. 
Sin embargo, nada de eso tendrá ningún valor ni perdurará si no mantenemos la humildad, motivación y dedicación en nuestra tarea cotidiana, elementos esenciales en toda empresa humana. De aquí en más habrá mayores desafíos y para mantener nuestra misión será necesario redoblar los esfuerzos y sostener el convencimiento de poder ofrecer una revista de la mejor calidad posible...".

Han pasado exactamente cuatro años y ahora otra vez tenemos la gran alegría de poder comunicarles que en nuestra revista estamos dando el primer paso de un cambio que también muy probablemente será histórico y que asimismo, estimamos que marcará otro hito en los algo más de 80 años de Archivos.

Desde el presente número comenzamos con la publicación en la versión electrónica de los artículos originales traducidos al idioma inglés. Hasta el momento actual la presencia de Archivos en Pubmed se efectiviza mediante los resúmenes en inglés. El lograr que se publique el texto completo en ese idioma, que sin duda es el que rige la literatura científica, será a nuestro criterio un salto cualitativo enorme. La difusión de los artículos se incrementará marcadamente y esto, además de lo que significa en sí mismo, contribuirá también a que aumenten los trabajos que recibimos para su evaluación, no solo en su número sino también en su procedencia.
Decimos que este es un primer paso porque iremos progresivamente traduciendo editoriales, comentarios y otros tipos de artículos. Nuestro deseo, de cumplirse las expectativas que esta iniciativa despierta, es que en algún momento lleguemos a traducir toda la revista, aunque eso dependerá de múltiples factores, algunos de los cuales podrían ser un obstáculo que esperamos pueda ser superado.

Deseo destacar que para alcanzar este logro fue muy importante el apoyo de la Comisión Directiva de la Sociedad que acogió con gran entusiasmo nuestra propuesta. Asimismo, la labor de todo el grupo de editores fue esencial en la ardua tarea que demandó esta iniciativa.

Será necesario continuar con los principios que señalé previamente, mantener la humildad y motivación, redoblar los esfuerzos y estar convencidos que la mayoría de las cosas que emprendemos y alcanzamos en la vida siempre son pasibles de mejorarlas.

José M Ceriani Cernadas
Editor
jceriani@sap.org.ar

http:/ /dx.doi.org/10.5546/aap.2012.283 